\title{
Metallic Ferromagnetism - an Electronic Correlation Phenomenon
}

\author{
D. Vollhardt ${ }^{1}$, N. Blümer ${ }^{1, *}$, K. Held ${ }^{2}$, and M. Kollar ${ }^{3}$ \\ 1 Theoretical Physics III, Center for Electronic Correlations and Magnetism, \\ University of Augsburg, 86135 Augsburg, Germany \\ 2 Dept. of Physics, Princeton University, Princeton, NJ 08544, USA \\ 3 Dept. of Physics, Yale University, P. O. Box 208120, New Haven, CT 06520-8120, \\ USA
}

\begin{abstract}
New insights into the microscopic origin of itinerant ferromagnetism were recently gained from investigations of electronic lattice models within dynamical meanfield theory (DMFT). In particular, it is now established that even in the one-band Hubbard model metallic ferromagnetism is stable at intermediate values of the interaction $U$ and density $n$ on regular, frustrated lattices. Furthermore, band degeneracy along with Hund's rule couplings is very effective in stabilizing metallic ferromagnetism in a broad range of electron fillings. DMFT also permits one to investigate more complicated correlation models, e.g., the ferromagnetic Kondo lattice model with Coulomb interaction, describing electrons in manganites with perovskite structure. Here we review recent results obtained with DMFT which help to clarify the origin of band-ferromagnetism as a correlation phenomenon.
\end{abstract}

\section{Introduction}

Since the Curie temperature of ferromagnetic metals like iron, cobalt, and nickel is of the order of electrostatic energies in solids, i.e., is much higher than the interaction energy of the electron spins, itinerant ferromagnetism is expected to be the result of the interplay between the ordinary spin-independent Coulomb interaction and the kinetic energy of the electrons, in combination with the Pauli principle [1,2,3]. As such it is one of the fundamental cooperative phenomena in condensed matter physics.

Until recently the theory of itinerant ferromagnetism was investigated by two essentially separate communities, one using model Hamiltonians in conjunction with many-body techniques (or even rigorous mathematical methods [4.5.6.6]), the other employing density functional theory (DFT) [7,8]. DFT and its local density approximation (LDA) have the advantage of being ab initio approaches which do not require empirical parameters as input. Indeed, they are highly successful techniques for the calculation of the electronic structure of real materials. However, in practice DFT/LDA is restricted in its ability to describe strongly correlated materials. Here, the model Hamiltonian approach is more general and powerful since there exist systematic theoretical methods to investigate the many-electron problem with increasing accuracy. Nevertheless, the uncertainty in the choice of the model parameters and the technical complexity of the correlation problem itself prevent the model Hamiltonian approach from being a 
flexible or reliable enough tool for studying real materials. The two approaches are therefore complementary.

Originally the one-band Hubbard model was introduced to gain insight into the origin of metallic ferromagnetism [9, 10,11. However, even for this simplest possible microscopic model answers are not easily obtained since in general metallic ferromagnetism occurs at intermediate couplings and off half filling [3, 12]. Thus, this cooperative phenomenon belongs to the class of problems where standard perturbation techniques are not applicable. In particular, weakcoupling theories or renormalization group approaches 13] which are so effective in detecting instabilities with respect to antiferromagnetism or superconductivity meet with limited success here. In general, non-perturbative investigations are required.

During the last couple of years significant progress was made in the theoretical understanding of the microscopic foundations of metallic ferromagnetism. They were made possible mainly by the development (i) of new analytic approaches, such as the mathematical methods used to investigate flat-band ferromagnetism [6] and its extensions [14,15] as well as dynamical mean-field theory (DMFT) [16,17,18], (ii) of new numerical techniques, such as the density matrix renormalization group (DMRG) which yields precise results in $d=1$ [19], and (iii) of new comprehensive approximation schemes such as the multiband Gutzwiller wave function [20], or the new ab initio computational scheme LDA+DMFT 21,22,23 which merges conventional band structure theory (LDA) with a comprehensive many-body technique (DMFT).

In this paper the insights gained with the help of the DMFT will be reviewed. After a short introduction into this approach (Section 2), the microscopic conditions for metallic ferromagnetism in the one-band Hubbard model (Section 3) and in the case of the orbitally degenerate model (Section 4) are explained and the differences discussed. Furthermore, the physics of itinerant ferromagnetism in more complicated models, e.g., the ferromagnetic Kondo lattice model with Coulomb correlations for manganites with perovskite structure, is analyzed. A conclusion and outlook (Section 5) closes the presentation.

\section{Dynamical Mean-Field Theory}

During the last decade a new many-body approach was developed which is especially well-suited for the investigation of correlated electronic systems with strong local interactions - the dynamical mean-field theory (DMFT) 24]. It becomes exact for $d=\infty$, i.e., for lattices with coordination number $Z=\infty$ [16. Why is the investigation of this limit useful and interesting? The answer is that already in $d=3$ the coordination number of regular lattices, such as the fcc lattice, is quite large $\left(Z_{\mathrm{fcc}}=12\right)$. It is therefore quite natural to view $Z$ as a large number, and to consider the limit $Z \rightarrow \infty$ as a starting point [16]. To obtain a meaningful model in this limit one has to scale the NN hopping amplitude in the kinetic energy (see below) as $t=t^{*} / \sqrt{Z}$ (in the following we set $t^{*}=1$ and thereby fix the energy scale). Then one obtains a purely local 
theory where the self-energy $\Sigma_{\mathbf{k}}(\omega)$ becomes $\mathbf{k}$ independent and where the propagator $G(\mathbf{k}, \omega)=G^{0}(\mathbf{k}, \omega-\Sigma(\omega))$ may be represented by the non-interacting propagator at a shifted frequency [24]. The dynamics of the quantum mechanical correlation problem is then fully included. That is why this theory is referred to as "dynamical mean-field theory" (DMFT). We note that due to the local nature of the theory there is no short-range order in position space.

Within DMFT the electronic lattice problem is mapped onto an effective selfconsistent single-impurity Anderson model 24]. DMFT is a non-perturbative, thermodynamically consistent theoretical framework within which the dynamics of correlated lattice electrons with local interaction can be investigated at all coupling strengths. This is of essential importance for problems like bandferromagnetism or the metal-insulator transition [25] which set in at a Coulomb interaction strength comparable to the electronic band width.

In DMFT the information about the lattice or the dispersion of the system under investigation enters only through the density of states (DOS) $N^{0}(E)$ of the non-interacting particles, unless there is long-range order with broken translational symmetry of the lattice as in the case of antiferromagnetism. In finite dimensions, e.g., $d=3$, DMFT is then an approximation - usually an excellent one as is manifested by the plethora of results obtained within the last decade [24. DMFT is presently the only comprehensive, thermodynamically consistent computational scheme which allows one to investigate the dynamics of correlated lattice electrons on all energy scales.

Due to its equivalence to an Anderson impurity problem a variety of approximative techniques have been employed to solve the complicated DMFT equations, such as the iterated perturbation theory (IPT) [26] and the noncrossing approximation (NCA) 27, as well as numerically exact techniques like quantum Monte-Carlo simulations (QMC) [28], exact diagonalization (ED) [29], or the numerical renormalization group (NRG) [30]. However, NRG cannot yet be used to solve the DMFT equations for multi-band models.

In the present paper all DMFT results were obtained by QMC. This is a particularly well-tested and reliable, albeit computer-expensive method which may be employed down to temperatures $T \sim 10^{-2} W$ (W: band width) and at not too strong interactions.

\section{The One-Band Hubbard Model}

The one-band Hubbard model

$$
H_{\mathrm{Hub}}=-\sum_{i, j, \sigma} t_{i j}\left(c_{i \sigma}^{\dagger} c_{j \sigma}+\text { h.c. }\right)+U \sum_{i} n_{i \uparrow} n_{i \downarrow}
$$

is the simplest lattice model for correlated electrons, and was originally proposed to understand metallic ferromagnetism in $3 d$ transition metals [9, 10,11. Here, $t_{i j}$ is a general hopping matrix element between sites $i$ and $j$. In the past the kinetic energy in (11) was usually restricted to nearest-neighbor (NN) hopping; then it is useful to divide the underlying lattices into bipartite and non-bipartite ones. 


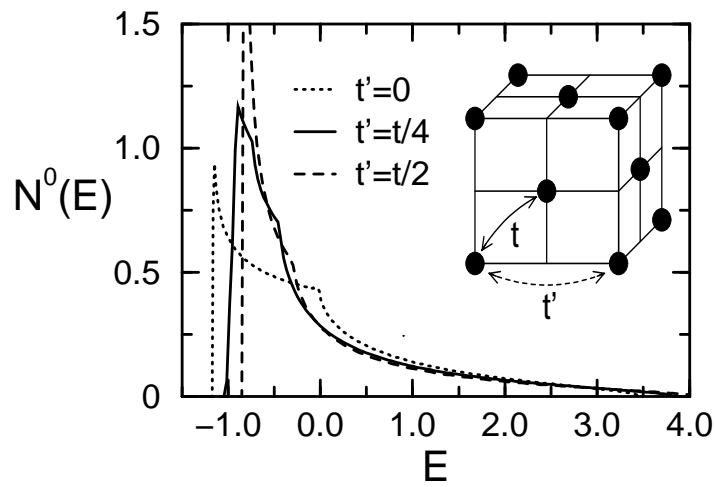

Fig. 1. DOS of noninteracting electrons on a fcc lattice in $d=3$ with and without additional NNN hopping $t^{\prime}$.

This distinction ceases to be useful if, in addition to NN hopping $t$, longer-range hopping, in particular next-nearest-neighbor (NNN) hopping $t^{\prime}$ is considered.

The Hubbard model is characterized by a purely local interaction term which is completely independent of the lattice structure and dimension. Therefore in this model the kinetic energy of the electrons (i.e., the dispersion) and the structure of the lattice are very important for the stability of metallic ferromagnetism. This is well-known from the approximate investigations by Gutzwiller [9], Hubbard [10], Kanamori [11] and Nagaoka 31], and has recently been confirmed, and made precise, by detailed investigations [6, 32, 15, 14, 17, 18].

\subsection{Routes to Ferromagnetism}

On bipartite lattices the $t^{\prime}$-hopping term destroys the antiferromagnetic nesting instability at small $U$ (see, for example [33]) by shifting spectral weight to the band edges and thereby introducing an asymmetry into the otherwise symmetric DOS. It will be shown below that a high spectral weight at the band edge (more precisely: the lower band edge for $n<1$ ) minimizes the loss of kinetic energy of the overturned spins in the magnetic state and is hence energetically favorable. Therefore frustrated, i.e., non-bipartite lattices, or bipartite lattices with frustration due to further-range hopping (e.g., $t^{\prime} \neq 0$ ) support the stabilization of metallic ferromagnetism.

The fcc lattice is an example of a frustrated lattice in $d=3$. The corresponding DOS of the non-interacting particles is shown in Fig. 1. Switching on an additional NNN hopping $t^{\prime}$ further increases the spectral weight at the lower band edge. For $t^{\prime}=t / 2$ one even obtains a square-root-like divergence. To understand why a high spectral weight at the band edge is favorable for the kinetic energy we first consider the case $U=0, n<1[12]$. Let us consider a flat, symmetric DOS. The fully polarized state is obtained by inverting the spin of the down electrons, which due to the Pauli principle have to occupy higher energy 
states. Counting the energy from the lower band edge the Fermi energy of the polarized state, $\mu_{\uparrow}$, is seen to be twice that of the unpolarized state. This should be contrasted with a DOS having large spectral weight at the lower band edge. Here the Fermi level of the polarized state is not so strongly shifted upwards, i.e., fewer high energy states are populated, which is clearly energetically favorable. The energy difference between the fully polarized state and the unpolarized state

$$
\Delta E=\left[\int_{-W_{1}}^{\mu_{\uparrow}}-2 \int_{-W_{1}}^{\mu}\right] d E N^{0}(E) E
$$

must become negative for the ferromagnetic state to be stable. Of course, in the non-interacting case one has $\Delta E>0$ 惯. Nevertheless, even for $U=0, \Delta E$ attains its lowest value for a DOS with peaked spectral weight at the lower band edge for all $n<1$ [12]. To show that $\Delta E<0$ for $U>0$ requires a reliable calculation of the energy of the correlated paramagnet. Indeed, this is one of the central, most difficult problems of electronic correlation theory. It should be noted that the above discussion concerning the shape of the DOS goes beyond the well-known Stoner criterion which predicts a ferromagnetic instability of the paramagnet for $U$ equal to the inverse of the DOS precisely at the Fermi level.

\section{2 $\quad$ Frustrated Lattices}

Since metallic ferromagnetism is an intermediate-coupling problem purely analytic approaches alone cannot provide sufficient information, in particular in dimensions $d>1$. In this situation the development of new numerical techniques during the last few years was of crucial importance for progress in this field. In particular, for one-dimensional systems the DMRG [19], and for highdimensional systems the DMFT have led to important insights. Here we restrict ourselves to results obtained by DMFT.

By a suitable generalization of the dispersion relation in three dimensions, frustrated lattices like the fcc lattice can be defined in any dimension, in particular in $d=\infty$ [34]. With the proper scaling of the hopping term (see above) the non-interacting DOS of the generalized $f c c$ lattice in $d=\infty$ is obtained as:

$$
N_{g f c c}^{0}(E)=e^{-(1+\sqrt{2} E) / 2} / \sqrt{\pi(1+\sqrt{2} E)}
$$

It has a strong square-root divergence at the lower band edge, $-1 / \sqrt{2}$, and no upper band edge.

Investigations of the stability of metallic ferromagnetism on fcc-type lattices within DMFT, in combination with finite-temperature QMC techniques to solve the DMFT equations, were first performed by Ulmke [35]. To detect a ferromagnetic instability one calculates the temperature dependence of the uniform static susceptibility $\chi_{F}$ from the corresponding two-particle correlation function [36]. For $N_{g f c c}^{0}(E)$ and at an intermediate interaction strength of $U=4$ the ferromagnetic response is found to be strongest around quarter filling $(n \simeq 0.5)$. The susceptibility $\chi_{F}$ is seen to obey a Curie-Weiss law (Fig. 2). Thus the Curie temper- 


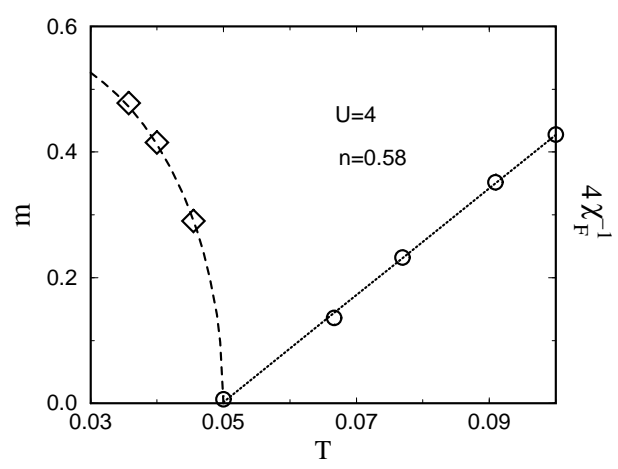

Fig. 2. Magnetization $m$ (diamonds) and inverse ferromagnetic susceptibility $\chi_{F}^{-1}$ (circles; note the factor of 4 in the definition) as calculated by DMFT for the one-band Hubbard model with DOS (3) at $U=4$ and $n=0.58$. Error-bars are of the size of the symbols or smaller. The value of $\chi_{F}^{-1}$ at $T=0.05$ is a data point, not an extrapolation. The dotted line is a linear fit to $\chi_{F}^{-1}$, the dashed line a fit with a Brillouin function to $m$ 35.

ature $T_{c}$ can be safely extrapolated from the zero of $\chi_{F}^{-1}$. For $n=0.58$ this leads to a value of $T_{c}=0.051(2)$. Below $T_{c}$ the magnetization $m$ (a one-particle quantity determined by the local propagator) grows rapidly, reaching more than $80 \%$ of the fully polarized value $\left(m_{\max }=n=0.58\right)$ at the lowest temperature $(30 \%$ below $\left.T_{c}\right)$. The three data points $m(T)$ in Fig. 2 are consistent with a Brillouin function with the same critical temperature of $T_{c}=0.05$ and an extrapolated full polarization at $T=0$. (We note that a saturated ground-state magnetization is also consistent with the single-spin-flip analysis of the fully polarized state by Uhrig [17). So one finds simultaneously a Curie-Weiss-type static susceptibility with Brillouin-function-type magnetization, and a non-integer magneton number, in qualitative agreement with experiments on $3 d$ transition metals. In the past, these features were attributed to seemingly opposite physical pictures: the former to localized spins, and the latter to itinerant electrons. However, this was only because of the use of oversimplified, static mean-field-type theories like Weiss mean-field theory (for spin models) and Hartree-Fock (for electrons). Here we see that these properties are natural features of correlated electronic systems, which are generated by the quantum dynamics of the many-body problem. Within DMFT this "paradoxical" behavior of band ferromagnets is resolved without difficulty.

Collecting the values of $T_{c}(U, n)$ obtained by $\chi_{F}^{-1}\left(T_{c}, U, n\right)=0$, the boundaries between the ferromagnetic and the paramagnetic phases are determined. Thereby one can construct the $T$ vs. $n$ phase diagram for different values of $U$. The region of stability increases with $U$ [35].

To make contact with $d=3$ one can use the corresponding fcc DOS shown in Fig. 1. For $t^{\prime}=0$ no instability is found at temperatures accessible to QMC. However, already a small contribution of $t^{\prime}$-hopping (which is present in any real system) is enough to stabilize a large region of metallic ferromagnetism in 


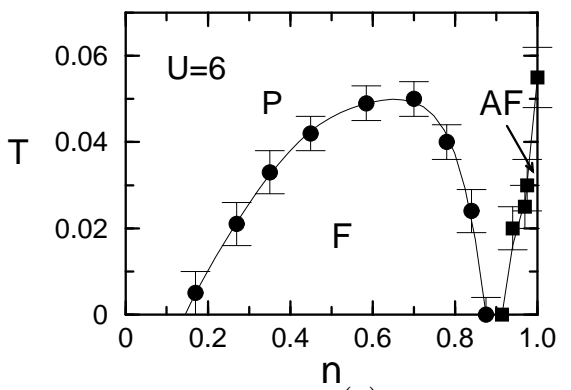

(a)

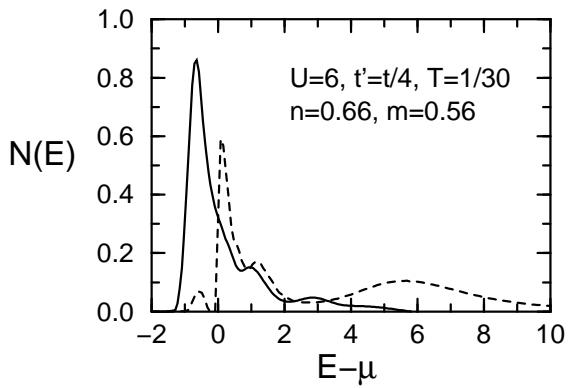

(b)

Fig. 3. (a) $T$ vs. $n$ phase diagram of the Hubbard model as obtained within DMFT for the DOS corresponding to a three-dimensional fcc lattice with NN hopping $t^{\prime}=t / 4$ (see Fig. 而); (b) DOS of the interacting electrons in the ferromagnetic phase of (a), solid line: majority spin, dashed line: minority spin [35].

addition to an antiferromagnetic phase (which is absent in $d=\infty$ ), close to half filling (Fig. 3a) [35]. This shows the strong and subtle dependence of the stability on the dispersion and the distribution of spectral weight in the DOS. By developing increasingly refined schemes of iterated perturbation theory to solve the DMFT equations Nolting and collaborator [37] obtained $T$ vs. $n$ phase diagrams which reproduce the QMC results remarkably well.

We note that the maximal transition temperature is $T_{c}^{\max }=0.05 \simeq 500 \mathrm{~K}$ for a band width $W=4 \mathrm{eV}$. This is well within the range of real transition temperatures, e.g., in nickel.

So far we only argued on the basis of the shape of the DOS of the non-interacting electrons, $N^{0}(E)$. On the other hand the interaction will renormalize the band and relocate spectral weight. Therefore it is not a priori clear at all whether the arguments concerning the kinetic energy discussed in subsection 3.1 still hold at finite $U$. We note that this effect is also not taken into account in any Stoner theory. To settle this point one may calculate the DOS of the interacting system, $N(E)$, by employing the maximum entropy method for analytic continuation. In Fig. 3b we show $N(E)$ obtained with $N_{g f c c}^{0}(E)$ and the parameter values used to calculate Fig. 2. Clearly the ferromagnetic system is metallic since there is appreciable weight at the Fermi level $(E=\mu)$. Furthermore, the spectrum of the majority spins is seen to be affected only slightly by the interaction, the overall shape of the non-interacting DOS being almost unchanged (the magnetization is quite large $(m=0.56$ at $n=0.66)$ and hence the electrons in the majority band are almost non-interacting). This implies that the arguments concerning the distribution of spectral weight in the non-interacting case and the corresponding kinetic energy are even applicable to the polarized, interacting case. The spectrum of the minority spins is slightly shifted to higher energies and has a pronounced peak around $E-\mu \simeq U=6$.

From Fig. 3b we find the exchange splitting $\Delta$ between the majority and minority bands to be $\Delta=0.8$. Comparing the quantity $U m(=3.4$ in the 


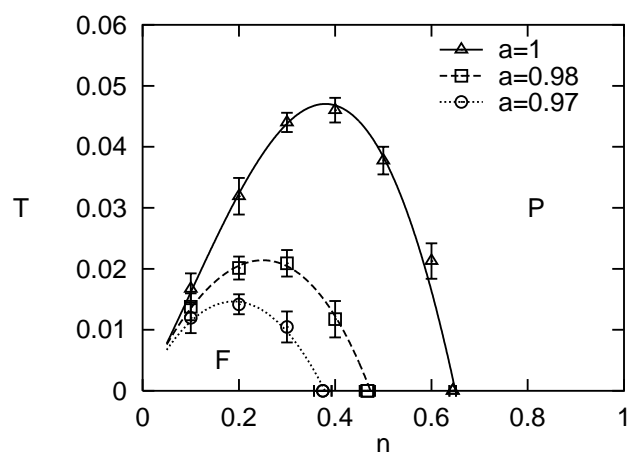

(a)

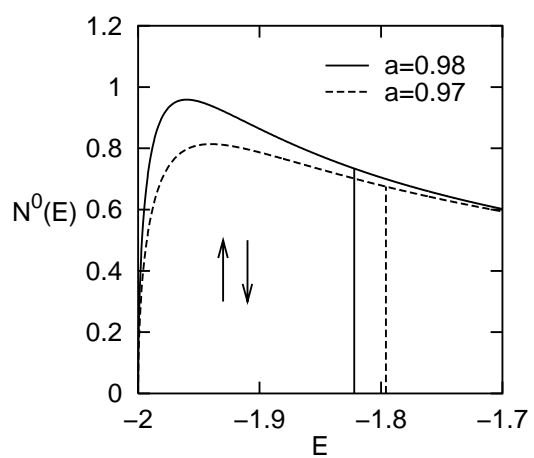

(b)

Fig. 4. (a) $T$ vs. $n$ phase diagram of the Hubbard model as obtained within DMFT; (b) corresponding shapes of the non-interacting DOS; Fermi energies for $n=0.3$ are indicated by vertical lines 38.

present case) with $\Delta$ and $T_{c}$ we find a characteristic hierarchy of energy scales:

$$
U m>\Delta \gg T_{c} .
$$

This is very different from results obtained by Stoner theory or effective oneparticle theories like LDA where all three quantities are essentially equal. The generation of small energy scales is a genuine correlation effect.

To study the influence of the distribution of spectral weight on the stability of ferromagnetism within the DMFT systematically, Wahle et al. 38 recently solved the DMFT equations with a tunable model DOS,

$$
N^{0}(E)=c \frac{\sqrt{D^{2}-E^{2}}}{D+a E}
$$

with $c=\left(1+\sqrt{1-a^{2}}\right) /(\pi D)$ and half-band width $D \equiv 2$. Here $a$ is an asymmetry parameter which can be used to change the DOS continuously from a symmetric, Bethe lattice DOS $(a=0)$ to a DOS with a square-root divergence at the lower band edge $(a=1)$, corresponding to a fcc lattice with $t^{\prime}=t / 4$ in $d=3$ (Fig. 1). The strong dependence of the stability of metallic ferromagnetism on the distribution of spectral weight is shown in Fig. Aa. Already a minute increase in spectral weight near the band edge of the non-interacting DOS, obtained by changing $a$ from 0.97 to 0.98 (see Fig. $4 \mathrm{~b}$ ) is enough to almost double the stability region of the ferromagnetic phase. Obermeier et al. [39] found ferromagnetism even on a hypercubic, i.e., bipartite, lattice, but only at very large $U$ values $(U>30)$.

The importance of genuine correlations for the stability of ferromagnetism is apparent from Fig. 5 , where the DMFT results are compared with Hartree-Fock theory [38]. The quantum fluctuations, absent in Hartree-Fock theory, are seen to reduce the stability regime of ferromagnetism drastically. Spatial fluctuations (e.g., spin waves), absent in the DMFT, may reduce the stability regime further. 


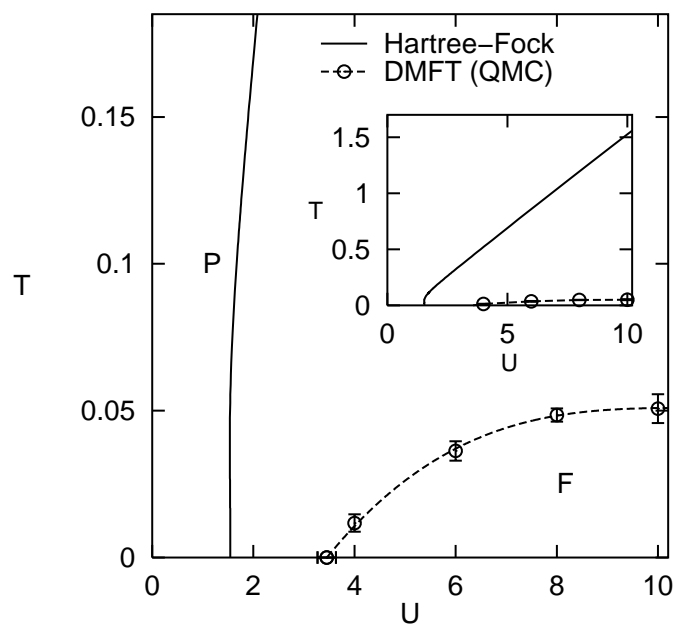

Fig. 5. $T$ vs. $U$ phase diagram for a strongly peaked DOS ( $a=0.98$, see Fig. $4 \mathrm{~b})$ at $n=0.4$ within DMFT (circles; dashed line is guide to the eyes only) in comparison with Hartree-Fock (solid line) 38].

\subsection{Additional Interactions}

In the one-band Hubbard model only the local interaction is retained. Thereby several interactions which naturally arise when the Coulomb interaction is expressed in Wannier representation are neglected. Even in the limit of a single band and taking into account only NN contributions, four additional interactions appear [10,40,41, 12]:

$$
\begin{aligned}
V_{1-\mathrm{band}}^{\mathrm{NN}}= & \sum_{\mathrm{NN}}\left[V n_{i} n_{j}+X \sum_{\sigma}\left(c_{i \sigma}^{\dagger} c_{j \sigma}+\text { h.c. }\right)\left(n_{i,-\sigma}+n_{j,-\sigma}\right)\right. \\
& \left.-2 F\left(\mathbf{S}_{i} \cdot \mathbf{S}_{j}+\frac{1}{4} n_{i} n_{j}\right)+F^{\prime}\left(c_{i \uparrow}^{\dagger} c_{i \downarrow}^{\dagger} c_{j \downarrow} c_{j \uparrow}+\text { h.c. }\right)\right] .
\end{aligned}
$$

Here the first term corresponds to a density-density interaction, the second term to a density-dependent hopping, and the fourth term describes the hopping of doubly occupied sites. In particular, the third term (with $F=F^{*} / Z>0$ )

$$
H_{F}=-2 \frac{F^{*}}{Z} \sum_{\mathrm{NN}} \mathbf{S}_{i} \cdot \mathbf{S}_{j}
$$

describes the direct ferromagnetic exchange between electrons on NN sites. It is this interaction which Heisenberg singled out in his original spin-model as the main source of ferromagnetism. It should be noted, however, that this interaction is present even when the electrons are free to move. The exchange interaction will be quite small, but nevertheless it favors ferromagnetic ordering in the most 


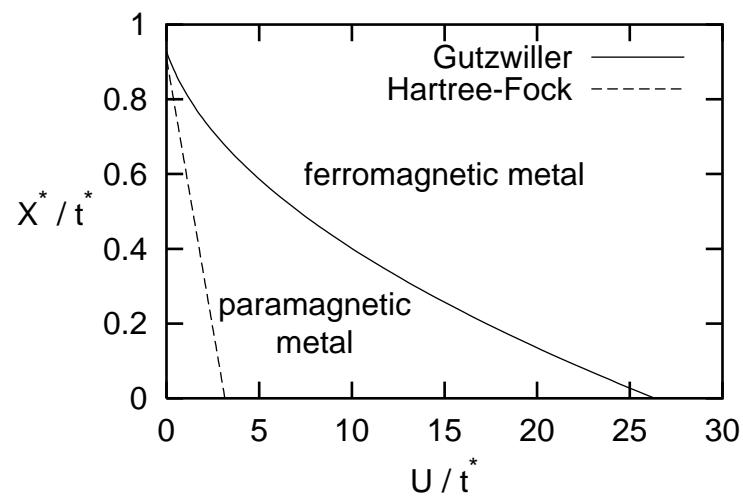

Fig. 6. Bond-charge interaction $X$ vs. $U$ phase diagram for the generalized model $H=H_{\mathrm{Hub}}+H_{X}$ within the Gutzwiller approximation for the semi-elliptical Bethe DOS at $n=0.9$.44.

obvious way. Hirsch 41] argued that this term is the main driving force for metallic ferromagnetism in systems like iron, cobalt, and nickel. Indeed, one can show rigorously that a next-neighbor direct exchange interaction, if only chosen large enough, easily triggers the ferromagnetic instability [42,12,38]. Hence the $\mathrm{NN}$ exchange may well be important for systems on the verge of a ferromagnetic instability.

Another term that can be of importance for the stabilization of ferromagnetism is the bond-charge interaction $\left(X=X^{*} / \sqrt{Z}\right)$

$$
H_{X}=\frac{X^{*}}{\sqrt{Z}} \sum_{\mathrm{NN} \sigma}\left(c_{i \sigma}^{\dagger} c_{j \sigma}+\text { h.c. }\right)\left(n_{i,-\sigma}+n_{j,-\sigma}\right) .
$$

It effectively gives rise to correlated hopping, i.e., the hopping amplitude of an electron now depends on the presence of electrons of the opposite spin polarization. The magnitude of $X$ has been estimated to be of order $0.1-1 \mathrm{eV} 10,40$, and hence $X$ may be comparable to the hopping $t$, although typically smaller than $U$. Hartree-Fock treatment of $H=H_{\mathrm{Hub}}+H_{X}$ shows that correlated hopping can lead to a spin-dependent band narrowing which may stabilize ferromagnetism [43. We note that in DMFT $H_{X}$ does not reduce to its Hartree-Fock contribution. The correlation effects introduced by $H_{X}$ were recently studied within the Gutzwiller approximation (GA) [44. The GA yields the exact evaluation of expectation values in terms of the Gutzwiller wave function in the limit of $d \rightarrow \infty$ [45.16] and goes beyond Hartree-Fock theory by including correlations explicitly. It was found that correlated hopping with $X>0$ can lower the critical value of $U$ for ferromagnetism considerably as shown in Fig. 6. Compared to Hartree-Fock theory the ferromagnetic region of the phase diagram is seen to be much reduced. Recently it was also shown how to incorporate $H_{X}$ into DMFT 46, at least in principle. It will be interesting to see how the GA results compare with future DMFT calculations for $H=H_{\mathrm{Hub}}+H_{X}$. 
Clearly, NN interactions may help to stabilize ferromagnetism. However, since the considerably larger Hubbard interaction $U$, together with a suitable kinetic energy, is already sufficient to trigger a ferromagnetic instability, the ferromagnetic exchange and bond-charge interactions appear to play only a secondary role.

\section{Band Degeneracy and Local Exchange}

Besides the NN Heisenberg exchange interaction another much larger exchange term is present in ferromagnets like iron, cobalt, and nickel, namely the local exchange between electrons in different orbitals on the same lattice site. It has long been speculated that this exchange interaction, which is known to align electrons on isolated atoms (Hund's first rule), may also lead to ferromagnetism in the bulk, being transmitted by the kinetic energy [47]. The simplest model for this mechanism is the two-band Hubbard model with local exchange $F_{0}$ and Coulomb repulsion $V_{0}$ between two orbitals $\nu=1,2$ (Fig. 7):

$$
\begin{aligned}
H_{2 \text {-band }}= & -t \sum_{\mathrm{NN}, \sigma \nu} c_{i \nu \sigma}^{\dagger} c_{j \nu \sigma}+U \sum_{i \nu} n_{i \nu \uparrow} n_{i \nu \downarrow} \\
& +\sum_{i \sigma \sigma^{\prime}}\left(V_{0}-\delta_{\sigma \sigma^{\prime}} F_{0}\right) n_{i 1 \sigma} n_{i 2^{\prime} \sigma^{\prime}}-F_{0} \sum_{\sigma \neq \sigma^{\prime}} c_{i 1 \sigma^{\dagger}}^{\dagger} c_{i 1 \sigma^{\prime}} c_{i 2 \sigma^{\prime}}^{\dagger} c_{i 2 \sigma^{\prime}} .
\end{aligned}
$$

This two-band model [48] and its multi-band generalizations [49] were studied in considerable detail by various theoretical techniques [50]. Recent investigations [51] were triggered by the renewed interest in the electronic properties of transition metal oxides where the doubly degenerate $e_{g}$ bands of the $d$ electrons plays a very important role. At quarter filling (one electron per site; $n=1$ ), ferromagnetism can be understood by superexchange within strong-coupling perturbation theory (Fig. 8). But away from quarter (or half) filling, the model is much more difficult to treat. In this regime DMFT, solved by QMC, once more provides a powerful tool to investigate this model [52], at least, if the last term in (9), i.e., the spin-flip contribution of $F_{0}$, is neglected.

The calculated $T$ vs. $n$ phase diagram is shown in Fig. 9 for a symmetric Bethe DOS. Without Hund's rule coupling $F_{0}$ ferromagnetism is unstable for this DOS, at least at moderate values of $U$ (see Sec. 3.2 for the one-band model and [52] for the two-band model). However, when the Hund's rule exchange is included a metallic ferromagnetic phase is stabilized between an alternating (staggered) orbital ordered state at quarter filling and antiferromagnetism at half filling.

While orbital ordering at $n=1$ and antiferromagnetism at $n=2$ can be understood by superexchange, the mechanism for metallic ferromagnetism is more subtle. Of course, the virtual superexchange processes described above are still present. But the additional electrons doped to the $n=1$ system may now move freely between two singly occupied sites, i.e., are unhindered by the Coulomb repulsion $U$ or $V_{0}$. Furthermore, if the electrons on the two sites are 


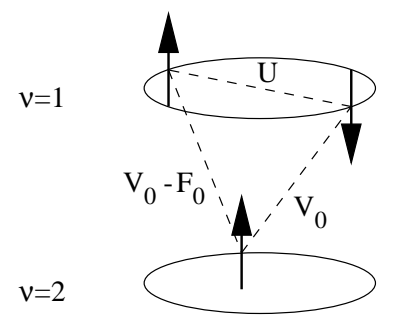

Fig. 7. Illustration of the local interactions (Hubbard $U$ and Hund's rule couplings $V_{0}, F_{0}$ defined in (9)) between electrons in a two-band model.

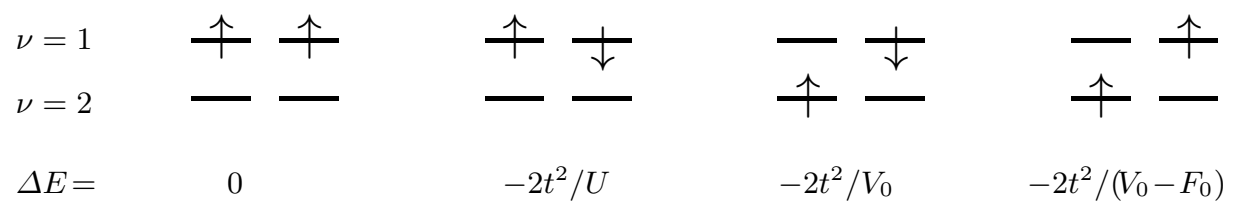

Fig. 8. Energy gain in second-order perturbation theory in $t$ for four configurations with two electrons on two sites. Most favorable is ferromagnetism combined with an alternating occupation of orbitals. Note, that the true ground state is not the last configuration above, i.e., $c_{i=1 \nu=1 \uparrow}^{\dagger} c_{22 \uparrow}^{\dagger}|0\rangle$, but the corresponding orbital singlet $\frac{1}{2}\left(c_{11 \uparrow}^{\dagger} c_{22 \uparrow}^{\dagger}-c_{12 \uparrow}^{\dagger} c_{21 \uparrow}^{\dagger}\right)|0\rangle$.

spin aligned they do not even have to spend the exchange energy $F_{0}$. Therefore a ferromagnetic alignment of the spins improves the kinetic energy of the electrons. This is essentially the concept of double exchange introduced by Zener [53] to explain ferromagnetism in manganites such as $\mathrm{La}_{1-x} \mathrm{Ca}_{x} \mathrm{MnO}_{3}$, and put on a firmer theoretical fundament by Anderson and Hasegawa [54.

In manganites, the cubic crystal field splits the five Mn $d$ orbitals into three $t_{2 g}$ and two $e_{g}$ orbitals. The former have a lower energy and hybridize less strongly with the $\mathrm{O} p$ orbitals. Thus, the three electrons within the $t_{2 g}$ orbitals can be approximately described by a localized spin $\mathbf{S}_{i}$, with the remaining $1-x$ electrons occupying the $e_{g}$ orbitals. If the Coulomb repulsion between $e_{g}$ electrons is taken into account one arrives at a correlated electron model for manganites:

$$
H=H_{2 \text {-band }}-2 J \sum_{\nu=1}^{2} \sum_{i} \mathbf{s}_{i \nu} \cdot \mathbf{S}_{i \nu} .
$$

Here, $H_{2 \text {-band }}$ is defined in (9), $\mathbf{s}_{i \nu}=\frac{1}{2} \sum_{\sigma \sigma^{\prime}} c_{i \nu \sigma^{\dagger}}^{\dagger} \tau_{\sigma \sigma^{\prime}} c_{i \nu \sigma^{\prime}}$ denotes the $e_{g}$ spin $(\tau$ : Pauli matrices), and $J$ is the local exchange between $t_{2 g}$ and $e_{g}$ electrons. Without Coulomb interaction $\left(U=V_{0}=F_{0}=0\right)$, the Hamiltonian (10) reduces to the ferromagnetic Kondo lattice model (KLM) which was investigated intensively in recent years 55. This model forms the theoretical basis for the double exchange mechanism: at $J \gg t$, the optimization of the kinetic energy of the $e_{g}$ electrons requires a ferromagnetic environment of $t_{2 g}$ spins. The KLM fails to describe the correct behavior for $x \lesssim 0.5$ since it does not penalize double occupations, i.e., two $e_{g}$ electrons on the same site. The suppression of double oc- 


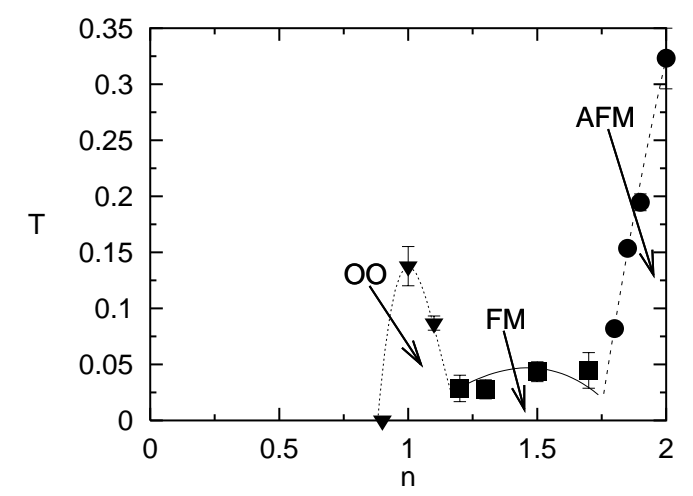

Fig. 9. $T$ vs. $n$ phase diagram of the two-band Hubbard model including antiferromagnetism (AF), ferromagnetism (FM), and orbital ordering (OO) for a Bethe DOS (total width $W=4$ ), $U=6, V_{0}=4$, and $F_{0}=2$ [52.

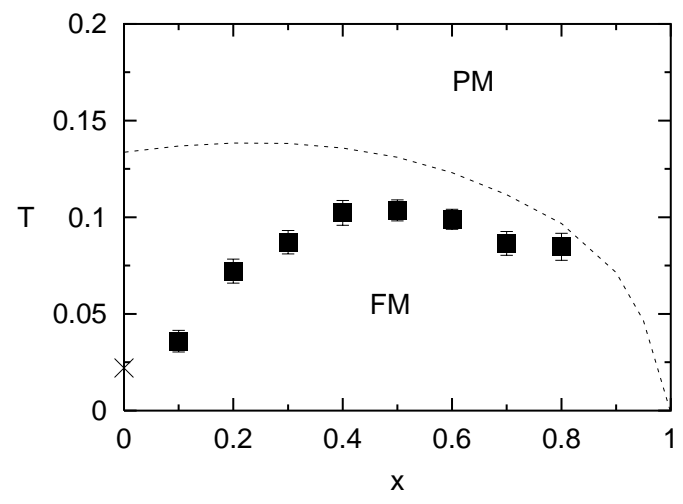

Fig. 10. Curie temperature $T_{c}$ for the phase transition from the paramagnetic (PM) to the ferromagnetic (FM) phase as a function of Ca doping $x(n=1-x)$. Dashed line: KLM with Bethe DOS (band width $W=2$ ) and Hund's rule coupling $J=3 / 2$; squares: correlated electron model (10) which also takes into account the Coulomb interaction between $e_{g}$ electrons $\left(U=8, V_{0}=6\right.$, and $\left.F_{0}=1\right)$; cross: Weiss mean-field theory (superexchange) for this model [56]. Note that, without the coupling to the $t_{2 g}$ spin, no ferromagnetism is observed in Fig. 9 for $n<1$ at similar interaction strengths.

cupations induced by the Coulomb repulsions $U$ and $V_{0}$ was investigated within DMFT in [56], and was shown to lead to a crossover from double exchange to superexchange. This results in a maximum in the Curie temperature (Fig. 10) in qualitative agreement with experiment [57. 


\section{Conclusion and Outlook}

In this paper we reviewed recent developments in our understanding of the origin of metallic ferromagnetism in the one-band Hubbard model and in banddegenerate models on the basis of the dynamical mean-field theory (DMFT). In the one-band Hubbard model metallic ferromagnetism is found to exist in a surprisingly large region of the $U$ vs. $n$ phase diagram. A stabilization of this phase at intermediate $U$ values requires a sufficiently large spectral weight near one of the band edges as is typical for frustrated (e.g., fcc-type) lattices which optimize the kinetic energy of the polarized state and, at the same time, frustrate the parasitic antiferromagnetic order. This finding, together with the results obtained for dimension $d=1$ 32, 15, 14, 19, finally establishes the stability of band ferromagnetism in the one-band Hubbard model on regular lattices and at intermediate values of the interaction $U$ and density $n$. Thereby one of the prominent questions of many-body theory in this field is finally answered.

By contrast, the origin of metallic ferromagnetism in the band-degenerate Hubbard model at intermediate $U$ values is not primarily a spectral weight effect but is already caused by moderately strong Hund's rule couplings . In this respect the origin of ferromagnetism in the orbitally degenerate model is more straightforward than that in the (less realistic) one-band case. Nevertheless, in the absence of orbital ordering the resulting magnetic phase diagrams are remarkably similar. The identification of a single main driving force for the stabilization of metallic ferromagnetism in the one-band and the band-degenerate Hubbard model, respectively, helps to differentiate between different mechanisms. However, in real systems these effects will tend to conspire, as is evident, for example, in nickel where an fcc lattice leads to a strongly asymmetric DOS and the band degeneracy brings with it Hund's rule couplings.

These insights also helped to understand the origin of itinerant ferromagnetism in more complicated models, e.g., the ferromagnetic Kondo lattice model with and without Coulomb correlations, which is employed to understand the physics of manganites with perovskite structure, like $\mathrm{La}_{1-x}(\mathrm{Sr}, \mathrm{Ca})_{x} \mathrm{MnO}_{3}$. It was found that in this model double exchange can explain ferromagnetism only for doping $x \gtrsim 0.5$. At lower values of $x$ the suppression of double occupations by the local Coulomb repulsion becomes more and more important and leads to a crossover from double exchange to superexchange. This results in a maximum of the Curie temperature in qualitative agreement with experiment.

As discussed in the Introduction the problem of metallic ferromagnetism was, until recently, investigated by two essentially separate communities - one using model Hamiltonians together with many-body techniques, the other employing density functional theory (DFT) in the local density approximation (LDA). In view of the individual power of LDA and the model Hamiltonian approach it is highly desirable to combine these techniques, thereby creating an enormous potential for all future $a b$ initio investigations of real materials, including, e.g., $f$-electron systems, Mott insulators and metallic ferromagnets. A combination of these two approaches had already been used to investigate band ferromagnetism some time ago [58. Recently, a fusion has started to emerge in new 
directions. One is the construction of multi-band Gutzwiller wave functions in combination with spin-density functional theory in the limit of large coordination numbers [20]. It was already successfully applied to ferromagnetic nickel, leading to significant improvements over LDA results. The other is the ab initio computational scheme LDA+DMFT [23] which was recently used to investigate transition metal oxides and to calculate the magnetic excitation spectrum of ferromagnetic iron [22]. Without doubt these and related methods [59] will rapidly develop into standard tools for future investigations of band ferromagnetism and other electronic correlation phenomena.

\section{Acknowledgments}

We thank M. Ulmke and J. Wahle for fruitful discussions. This work was supported in part by the Sonderforschungsbereich 484 of the Deutsche Forschungsgemeinschaft and by a Feodor-Lynen grant of the Alexander von HumboldtFoundation (KH).

\section{References}

* Present address: Institut für Physik, KoMa 337, Johannes Gutenberg-Universität, 55099 Mainz, Germany

1. N. W. Ashcroft, N. D. Mermin: Solid State Physics (Saunders College, Philadelphia 1976)

2. D. C. Mattis: The Theory of Magnetism I (Springer, Berlin 1988)

3. P. Fazekas: Lecture Notes on Electron Correlation and Magnetism (World Scientific, Singapore 1999)

4. E. H. Lieb, D. Mattis: Phys. Rev. 125, 164 (1962)

5. E. H. Lieb: in The Hubbard Model: Its Physics and Mathematical Physics, edited by D. Baeriswyl et al., p. 1, (Plenum, New York 1995)

6. A. Mielke: J. Phys. A 24, 3311 (1991); H. Tasaki: Phys. Rev. Lett. 69, 1608 (1992); A. Mielke, H. Tasaki: Commun. Math. Phys. 158, 341 (1993); H. Tasaki: Phys. Rev. Lett. 73, 1158 (1994); H. Tasaki: Phys. Rev. Lett. 75, 4678 (1995); H. Tasaki: Prog. Theor. Phys. 99, 489 (1998)

7. P. Hohenberg, W. Kohn: Phys. Rev. B 136, 864 (1964); W. Kohn, L. J. Sham: Phys. Rev. 140, 4A, A1133 (1965)

8. V. L. Moruzzi, J. F. Janak, A. R. Williams: Calculated Electronic Properties of Metals (Pergamon Press, New York 1978)

9. M. C. Gutzwiller: Phys. Rev. Lett. 10, 59 (1963)

10. J. Hubbard: Proc. Roy. Soc. London A 276, 238 (1963)

11. J. Kanamori: Prog. Theor. Phys. 30, 275 (1963)

12. For reviews see D. Vollhardt, N. Blümer, K. Held, M. Kollar, J. Schlipf, M. Ulmke: Z. Phys. B 103, 283 (1997); D. Vollhardt, N. Blümer, K. Held, M. Kollar, J. Schlipf, M. Ulmke, J. Wahle: Advances In Solid State Physics 38, p. 383 (Vieweg, Wiesbaden 1999)

13. R. Shankar: Rev. Mod. Phys. 66, 129 (1994); C. J. Halboth, W. Metzner: Phys. Rev. B 61, 7364 (2000); C. Honerkamp: Ph.D. Thesis, Diss. No. 13868, ETH-Zürich (2000) 
14. K. Penc, H. Shiba, F. Mila, T. Tsukagoshi: Phys. Rev. B 54, 4056 (1996)

15. P. Pieri, S. Daul, D. Baeriswyl, M. Dzierzawa: Phys. Rev. B 54, 9250 (1996)

16. W. Metzner, D. Vollhardt: Phys. Rev. Lett. 62, 324 (1989)

17. G. S. Uhrig: Phys. Rev. Lett. 77, 3629 (1996)

18. T. Hanisch, G. S. Uhrig, E. Müller-Hartmann: Phys. Rev. B 56, 13960 (1997)

19. S. Daul, R. Noack: Z. Phys. B 103, 293 (1997); S. Daul, R. Noack: Phys. Rev. B 58, 2635 (1998)

20. J. Bünemann, F. Gebhard, W. Weber: J. Phys. Condens. Matter 9, 7343 (1997); J. Bünemann, W. Weber, F. Gebhard: Phys. Rev. B 57, 6896 (1998); J. Bünemann, F. Gebhard, W. Weber: condmat/0006283, to be published in Foundations of Physics (2000)

21. V. I. Anisimov, A. I. Poteryaev, M. A. Korotin, A. O. Anokhin, G. Kotliar: J. Phys.: Cond. Matt. 9, 7359 (1997)

22. M. I. Katsnelson, A. I. Lichtenstein: Phys. Rev. B 57, 6884 (1998)

23. For an introduction into LDA+DMFT, see K. Held, I. A. Nekrasov, N. Blümer, V. I. Anisimov, D. Vollhardt: preprint cond-mat/00010395, to be published in Int. J. Mod. Phys. C

24. D. Vollhardt: Correlated Electron Systems, ed. by V. J. Emery (World Scientific, Singapore 1993); T. Pruschke, M. Jarrell, J. K. Freericks: Adv. Phys. 44, 187 (1995); A. Georges, G. Kotliar, W. Krauth, M. Rozenberg, Rev. Mod. Phys. 68, $13(1996)$

25. G. Moeller, Q. Si, G. Kotliar, M. Rozenberg, D. S. Fisher: Phys. Rev. Lett. 74, 2082 (1995); J. Schlipf, M. Jarrell, P. G. J. van Dongen, N. Blümer, S. Kehrein, Th. Pruschke, D. Vollhardt: Phys. Rev. Lett. 82, 4890 (1999); M. J. Rozenberg, R. Chitra, G. Kotliar: Phys. Rev. Lett. 83, 3498 (1999); R. Bulla: Phys. Rev. Lett. 83, 136 (1999)

26. A. Georges, G. Kotliar: Phys. Rev. B 45, 6479 (1992)

27. Th. Pruschke, D. L. Cox, M. Jarrell: Phys. Rev. B 47, 3553 (1993)

28. J. E. Hirsch, R. M. Fye, Phys. Rev. Lett. 56, 2521 (1986); M. Jarrell, Phys. Rev. Lett. 69, 168 (1992); M. Rozenberg, X. Y. Zhang, G. Kotliar, Phys. Rev. Lett. 69, 1236 (1992); A. Georges, W. Krauth, Phys. Rev. Lett. 69, 1240 (1992)

29. M. Caffarel, W. Krauth: Phys. Rev. Lett. 72, 1545 (1994)

30. R. Bulla, Phys. Rev. Lett. 83, 136 (1999); R. Bulla, T. Costi, D. Vollhardt: in preparation

31. Y. Nagaoka: Phys. Rev. 147, 392 (1966)

32. E. Müller-Hartmann: J. Low Temp. Phys. 99, 349 (1995)

33. W. Hofstetter, D. Vollhardt: Ann. Physik 7, 48 (1998)

34. E. Müller-Hartmann: Proc. V Symp. Phys. of Metals, ed. E. Talik and J. Szade, p.22 (Poland 1991)

35. M. Ulmke: Euro. Phys. J. B 1, 301 (1998)

36. M. Ulmke, V. Janiš, D. Vollhardt: Phys. Rev. B 51, 10411 (1995)

37. M. Potthoff, T. Herrmann, W. Nolting: Eur. Phys. J. B 4, 485 (1998); T. Wegner, M. Potthoff, W. Nolting, Phys. Rev. B 57, 6211 (1998); T. Herrmann, W. Nolting: J. Phys.: Condens. Matter 11, 89 (1999); W. Nolting: in Lectures on the Physics of Highly Correlated Electron Systems IV, AIP Conference Proceedings 527, p. 118 (Melville, New York 2000)

38. J. Wahle, N. Blümer, J. Schlipf, K. Held, D. Vollhardt: Phys. Rev. B 58, 12749 (1998)

39. T. Obermeier, T. Pruschke, J. Keller: Phys. Rev. B 56, R8479 (1997)

40. J. T. Gammel, D. K. Campbell: Phys. Rev. Lett. 60, C 71 (1988); D. K. Campbell, J. T. Gammel, E. Y. Loh Jr.: Phys. Rev. B 38, 12043 (1988) 
41. J. E. Hirsch: Phys. Rev. B 40, 2354 (1989); 9061 (1989); S. Tang, J. E. Hirsch: Phys. Rev. B 42, 771 (1990)

42. R. Strack, D. Vollhardt: Phys. Rev. Lett. 72, 3425 (1994); M. Kollar, R. Strack, D. Vollhardt: Phys. Rev. B 53, 9225 (1996)

43. J. Amadon, J. E. Hirsch: Phys. Rev. B 54, 6364 (1996)

44. M. Kollar, D. Vollhardt: preprint cond-mat/0008015, to be published in Phys. Rev. B 63(2001)

45. W. Metzner, D. Vollhardt: Phys. Rev. Lett. 59, 121 (1987)

46. A. Schiller: Phys. Rev. B 60, 15660 (1999)

47. J. C. Slater: Phys. Rev. 49, 537 (1936); J. H. van Vleck: Rev. Mod. Phys. 25, 220 (1953)

48. L. M. Roth: Phys. Rev. 149, 306 (1966); K. I. Kugel', D. I. Khomskiǔ: Sov. Phys.JETP 37, 725 (1973); M. Cyrot, C. Lyon-Caen: J. Phys. C 36, 253 (1975); K.A. Chao, J. Spałek, A. M. Oleś: Phys. Stat. Sol. (b) 84, 747 (1977); W. Gill, D. J. Scalapino: Phys. Rev. B 35, 215 (1987); H. Hasegawa: Phys. Rev. B 56, 1196 (1997); D. Meyer, W. Nolting: J. Phys.: Condens. Matter 11, 5811 (1999)

49. A. M. Oleś: Phys. Rev. B 23, 271 (1981); G. Stollhoff, P. Thalmeier: Z. Phys. B 43, 13 (1981); A. M. Oleś: Phys. Rev. B 28, 327 (1983); A. M. Oleś, G. Stollhoff: Phys. Rev. B 29, 314 (1984); A. M. Oleś, G. Stollhoff: Europhys. Lett. 5, 175 (1988); W. Nolting, W. Borgieł: Phys. Rev. B 39, 6962 (1989); G. Stollhoff, A. M. Oleś, V. Heine: Phys. Rev. B 41, 7028 (1990); R. Frésard, G. Kotliar: Phys. Rev. B 56, 12909 (1997)

50. Another two-band model for ferromagnetism is the periodic Anderson model, see G. G. Reddy, D. Meyer, S. Schwieger, A. Ramakanth, W. Nolting: J. Phys: Condens. Matter 12, 7463 (2000); D. Meyer, W. Nolting: Phys. Rev. B 62, 5657 (2000); D. Meyer, W. Nolting: to be published in Eur. Phys. J. B (2000)

51. J. Kuei, R. T. Scalettar: Phys. Rev. B 55, 14968 (1997); J. E. Hirsch: Phys. Rev. B 56, 11022 (1997); M. Fleck, A. M. Oleś, L. Hedin: Phys. Rev. B 56, 3159 (1997); T. Okabe: Prog. Theor. Phys. 98, 331 (1997); P. Fazekas: condmat/0011354, to be published in Foundations of Physics (2000)

52. K. Held, D. Vollhardt: Euro. Phys. J. B 5, 473 (1998)

53. C. Zener: Phys. Rev. 82, 403 (1951)

54. P. W. Anderson, H. Hasegawa: Phys. Rev. 100, 675 (1955)

55. See for example, N. Furukawa: J. Phys. Soc. Jap. 63, 3214 (1994); E. MüllerHartmann, E. Dagotto: Phys. Rev. B 54, 6819 (1996); S. Yunoki, A. Moreo, E. Dagotto: Phys. Rev. Lett. 81, 5612 (1998); A. Moreo, S. Yunoki, E. Dagotto: Science 283, 2034 (1999); E. Dagotto, S. Yunoki, A. Moreo: in Physics in Manganites, ed. T. A Kaplan, S. D. Mahanti (Kluwer Academic/Plenum, New York 1999); N. Furukawa: in Physics in Manganites, ed. T. A Kaplan, S. D. Mahanti (Kluwer Academic/Plenum, New York 1999); S. Yunoki, J. Hu, A. L. Malvezzi, A. Moreo, N. Furukawa, E. Dagotto: Phys. Rev. Lett. 80, 845 (1998); J. van den Brink, D. I. Khomskiǔ: Phys. Rev. Lett. 82, 1016 (1999)

56. K. Held, D. Vollhardt: Phys. Rev. Lett. 84, 5168 (2000)

57. P. Schiffer, A. P. Ramirez, W. Bao, S.-W. Cheong: Phys. Rev. Lett. 75, 3336 (1995)

58. W. Nolting, W. Borgieł, V. Dose, Th. Fauster: Phys. Rev. B 40, 5015 (1989); W. Borgieł W. Nolting, M. Donath: Solid State Commun. 78, 825 (1989); W. Borgieł W. Nolting: Z. Phys. B 78, 241 (1990); W. Nolting, A. Vega, Th. Fauster: Z. Phys. B 96, 357 (1995)

59. T. Wegner, M. Potthoff, W. Nolting, Phys. Rev. B 61, 1386 (2000) 